\title{
Application of $\mathrm{Yb}, \mathrm{Er}$ :Glass laser radiation for active drug delivery at the treatment of onychomycosis
}

\author{
Andrey V. Belikov, Anastasia D. Tavalinskaya*, Sergey N. Smirnov, and Andrey N. Sergeev \\ ITMO University, 49 Kronverksky Pr., Saint Petersburg 197101, Russian Federation \\ * e-mail: ntavalin@gmail.com
}

\begin{abstract}
The paper discusses the active delivery of a drug (methylene blue solution) through the Er:YLF-laser-perforated nail plate using pulsed radiation of microsecond Yb,Er:Glass laser. Different methods of laser impact on liquid drug are considered: by focused and diverging laser beam. It was established that both discussed methods can provide sufficient dose of the drug for the treatment of onychomycosis. (C) 2019 Journal of Biomedical Photonics \& Engineering.
\end{abstract}

Keywords: laser; drug delivery; nail; hydrodynamic effects; treatment of onychomycosis; photodynamic therapy.

Paper \#3318 received 15 Jan 2019; revised manuscript received 15 Mar 2019; accepted for publication 23 Mar 2019; published online 30 Mar 2019. doi: 10.18287/JBPE19.05.010305.

\section{Introduction}

Drug delivery remains an important task, including in the treatment of one of the common pathologies of the nail - onychomycosis. Currently, the treatment of onychomycosis with the help of local drugs, including photodynamic preparations, is ineffective, since the low permeability of the nail plate limits the delivery of a therapeutically sufficient amount of the drug to the lesion. Solutions of methylene blue [1], radachlorin [2], photoditazine [3], etc. are often used as photodynamic preparations. The permeability of the nail plate can be increased using chemical (acids, alcohols, glycols, etc.), physical (microperforation, iontophoresis, sonophoresis, electroporation; ultrasonic and laser drug delivery) and mechanical (injection, nail plate removal or mechanical perforation) [4-12] methods. Delivery can be passive or active. The latter occurs as a result of any external impact.

To accelerate the delivery of drugs under the nail plate, an array of microholes is used, created, among other methods, as a result of exposure to laser radiation. Amount of the drug delivered increases in proportion to the increase in the number of microholes in the array. In the paper [11] $\mathrm{CO}_{2}$-laser-produced microperforation of the nail plate allowed to target the "Exelderm" in the form of liquid solution and cream with $1 \%$ Sulconazole nitrate concentration to the depth of more than $370 \mu \mathrm{m}$. In Ref. [13] the fractional $\mathrm{CO}_{2}$-laser assisted drug delivery for effective and safe treatment of onychomycosis is discussed. It is shown that tazarotene $0.1 \%$ gel and tioconazole $28 \%$ solution are effective in onychomycosis treatment, moreover, the thermal impact of $\mathrm{CO}_{2}$-laser radiation is suggested to play a positive role in fungi destruction. For efficient microperforation, Er:YLF laser radiation [14] can also be used.

Unfortunately, drugs have an extremely low penetration rate in passive delivery including through an array of microholes. The passive drug delivery of wateralcohol methylene blue (MB) solution requires about 60-120 s, and water solution of MB do not penetrate through microholes with diameter 220-350 $\mu \mathrm{m}$ [15].

The impact of pulsed radiation of near and middle IR lasers can initiate hydrodynamic effects in liquids. Experimental studies shown that irradiation of water with pulses of Er:YAG [15], Er:YSGG [17], Ho:YAG [17,18] and Yb,Er:Glass [19-21] lasers, whose wavelengths lie near its intense absorption bands, can stimulate the appearance of thermoelastic waves and cavitation processes in water, accompanied by the generation of pressure transients. In Refs. [19-21] the hydrodynamic effects initiated in water by microsecond pulses of Yb,Er:Glass laser with a wavelength of $1.54 \mu \mathrm{m}$ are considered in detail. A significant influence of these effects on the removal efficiency of soft tissue was established [20, 22].

The rate of penetration of drugs into biological tissue can be increased due to the excitation of laserinduced hydrodynamic effects [23, 24]. In Ref. [24], a method used laser-induced hydrodynamic shock waves was proposed and investigated for the rapid delivery of solid microparticles, nanoparticles and fluids into tissues through multiple microchannels created as a result of Er:YAG laser fractional microablation. Among 
other things, it was shown that the active delivery of $\mathrm{ZrO}_{2}$ particles to microchannels induced by hydrodynamic shock waves generated by Er:YAG laser pulses performed at very high speed.

Unfortunately, the method combining effective laser microperforation and effective active laser delivery of drugs for the treatment of onychomycosis is not still described in literature. This stimulated the authors of this work to study the active delivery of a photodynamic drug $(0.25 \%$ methylene blue aqueous solution) to the nail bed through a single microhole (created in the nail plate by Er:YLF laser pulses) as a result of the excitation of hydrodynamic processes in the drug solution by the radiation of $\mathrm{Yb}$,Er:Glass laser.

Thus, the purpose of the current study is to determine the possibility of active laser delivery of the aqueous solution of methylene blue through a single microhole in the nail plate in vitro, determining the dependence of the number of pulses required for active laser drug delivery and the mass of the drug penetrated under the nail plate on the thickness of the drug layer, with diverging and focused $\mathrm{Yb}$,Er:Glass laser radiation.

\section{Materials and methods}

In experiments in vitro, the samples used represented fragments of healthy nail plates of 7 volunteers aged 22-45 years (2 men and 5 women). Fragments were obtained as a result of mechanical cutting of the upper edge of the nail as it grows normally. A total of 250 samples were examined. Samples were stored outdoors at room temperature in a dark place for no more than 30 days. Before experiments, the samples were mechanically cleaned of dirt and washed with distilled water. The average thickness of the samples was $350 \pm 20 \mu \mathrm{m}$.

To produce microholes in the nail plate, Er:YLF laser radiation $(\lambda=2.81 \mu \mathrm{m})$ was used. The scheme of the experimental setup is shown in Fig. 1(a). The pulse duration of the Er:YLF laser was $270 \pm 10 \mu$ s (base) (Fig. 1(b)).

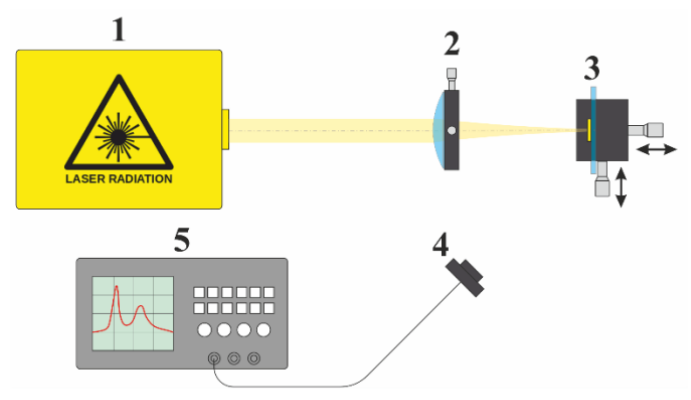

a
The laser pulse energy $(E)$ was $4.0 \pm 0.1 \mathrm{~mJ}$ at a pulse repetition frequency $(f)$ of $30 \mathrm{~Hz}$. The diameter of the laser beam on the surface of the nail plate was $220 \pm 15 \mu \mathrm{m}$. To control the shape and depth of the microholes, an optical microscopic registration of the appearance of laser-induced microdamages of the nail plate and the appearance of their longitudinal sections was carried out using an "Axio Scope A1" microscope (Carl Zeiss, Germany). A single through microhole with a diameter of $\sim 300 \mu \mathrm{m}$ was formed in the nail plate when exposed to 30 pulses of laser radiation. In Ref. [15] was obtained that at $300 \mu \mathrm{m}$ hole's diameter the rate of $0.25 \%$ alcohol $\mathrm{MB}$ solution penetration is closed to maximal. The further increase in hole's diameter influenced the penetration rate not significant.

Experimental setup for the study of the process of active delivery of the aqueous methylene blue solution through a single microhole in the nail plate is presented in Fig. 2(a).

The time structure of $\mathrm{Yb}$,Er:Glass laser pulse was obtained using the "FGA01" (Thorlabs, Inc., USA) InGaAs photodiode with $300 \mathrm{ps}$ rise time connected to oscilloscope through a $50 \Omega$ load. The pulses of Yb,Er:Glass laser radiation used for active delivery had a pronounced high-intensity "leading" spike (Fig. 2(b)), which makes it possible to amplify the excitation hydroacoustic effects [20]. The energy of Yb,Er:Glass laser pulse varied in the range from 10 to $100 \mathrm{~mJ}$, the pulse duration was $1.5 \pm 0.5 \mu$ s (base).

To visualize the penetration of the drug the paper with a density of $80 \mathrm{~g} / \mathrm{m}$ and a thickness of $100 \mu \mathrm{m}$ was placed under the sample. Coloration of the paper indicated the penetration of the drug through a microhole in the nail plate. The nail plate and paper were pressed against the glass substrate in such a way as to ensure tight contact between them. A $0.25 \%$ aqueous solution of methylene blue $(250 \mathrm{mg}$ of methylene blue powder per $100 \mathrm{~g}$ of distilled water) was used as a drug.

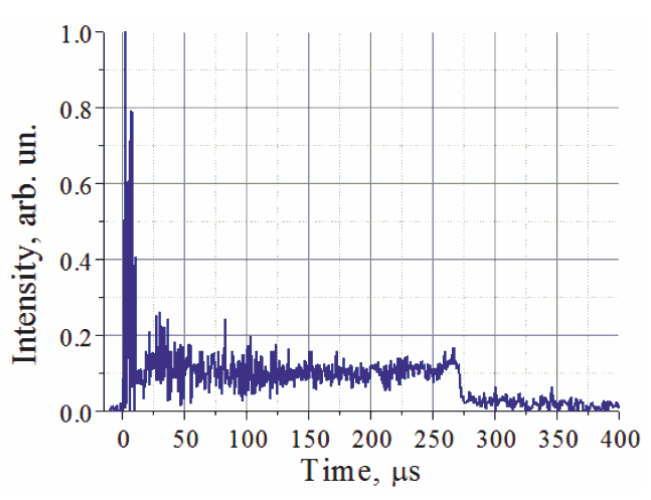

$\mathrm{b}$

Fig. 1 Scheme of the experimental setup for creating microholes in the nail plate (a): 1- Er:YLF laser; 2 - plano-convex lens $(\mathrm{F}=50 \mathrm{~mm}), 3$ - nail plate on a glass substrate; 4 - germanium photodetector, 5 - oscilloscope; Er:YLF laser pulse $(E=4 \mathrm{~mJ})(\mathrm{b})$. 


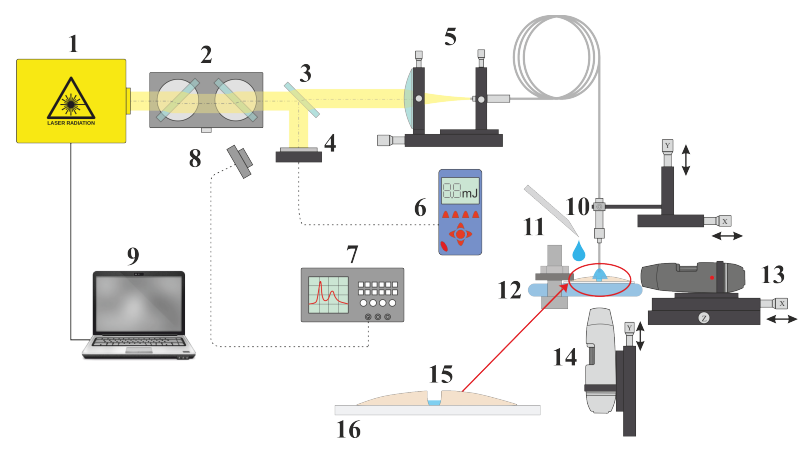

a

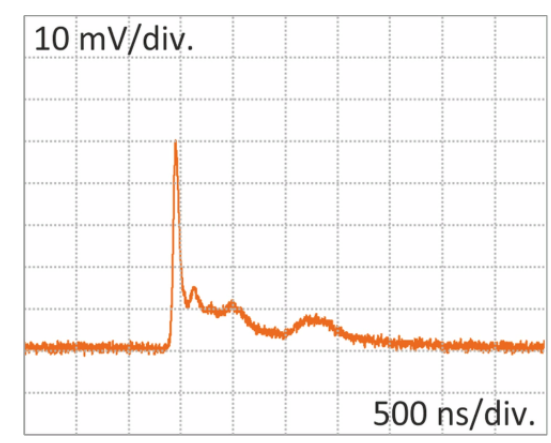

b

Fig. 2 Scheme of the experimental setup for investigating the process of active delivery of a methylene blue solution through a perforated nail plate (a): 1 - Yb,Er:Glass laser $(\lambda=1.54 \mu \mathrm{m}) ; 2-$ Fresnel attenuator; 3 - beam splitter; 4 -"PE50BF-C" sensor (Ophir Optronics Ltd., Israel); 5 - unit for coupling laser radiation into the optical fiber; 6 - energy meter "Nova II" (Ophir Optronics Ltd., Israel); 7 oscilloscope; 8 - InGaAs photodetector; 9 - computer; 10 - fiber output; 11 - drug injection needle (methylene blue solutions); 12 - glass substrate; 13 - "DTX 50" USB digital microscope (Levenhuk, Inc., USA); $14-$ "Prima Expert" digital USB-microscope (LOMO OJSC, Russia); 15 - nail plate; 16 - paper; Yb,Er:Glass laser pulse $(E=100 \mathrm{~mJ})(\mathrm{b})$.

This concentration is safe for the human body and effective against fungi and bacteria. Methylene blue is a widely used photosensitizer for photodynamic therapy of fungal diseases [1]. The choice of this drug is also because it has a rich blue color and is well visualized on the paper. A layer of the aqueous methylene blue solution was applied to the surface of the nail plate with a single microhole using a micropipette. The thickness of the drug (MB) layer $h$ was controlled by an optical method, while photographs of the nail plate without and with the drug layer were analyzed.

Active delivery was carried out in two ways: 1) by diverging $\mathrm{Yb}$,Er:Glass laser radiation from the output of quartz fiber $(N A=0.18, d=470 \mu \mathrm{m}$, the distal end of the fiber was placed at different distances from the drug solution surface); 2) by focused radiation of Yb,Er:Glass laser $(F=11 \mathrm{~mm}$, laser beam diameter $d_{b}=250 \pm 20 \mu \mathrm{m}$, focus on the surface of the nail plate).
The laser impact was implemented coaxially to the center of the microhole. The moment of paper coloration as a result of penetration of the drug under the nail plate was recorded using a digital USB microscope "Prima Expert" (LOMO OJSC, Russia). This moment corresponded to the "initialization" of the drug delivery. So, the fact of active drug delivery was detected by paper coloration. The experiment determined the number of laser pulses required for this "initialization".

The mass of the drug applied to the surface of the nail was determined by multiplying the drop volume by the density of the drug solution, which was taken equal to the density of water due to the low concentration of the dye. The mass penetrated under the nail plate with a single microhole was determined by weighing a piece of paper before and after the "initialization". For methylene blue, a dose of $5-10 \mathrm{mg} / \mathrm{cm}^{2}$ can be considered as therapeutically sufficient [25]. In the experiment, the dose of the drug penetrated under the nail plate was determined as the ratio of the mass of the drug penetrated during "initialization" to the area of a single microhole in the nail plate.

\section{Research results}

Using pulsed diverging Yb,Er:Glass laser radiation delivered via optical fiber whose distal end is located at a distance of $H=0.1 \mathrm{~mm}$ from the surface of drug drop applied to the nail plate it was possible to initiate the active drug delivery (detected by paper coloration) for $h$ up to $1 \mathrm{~mm}$ at pulse energies $E \geq 20 \mathrm{~mJ}$ (Fig. 3(a)).

In the studied range of $h(0.1 \div 1.0 \mathrm{~mm})$, except for the range $h=0.9 \div 1 \mathrm{~mm}$ for $E=20$ and $30 \mathrm{~mJ}$, an increase in $h$ resulted in a decrease in the number of pulses required for "initialization". The fastest "initialization" was obtained for $h=1.0 \mathrm{~mm}$ with $E=40 \mathrm{~mJ}$, the number of pulses required for "initialization" in this case was $N=1$.

The dependences of the mass of the drug (PM) penetrated through a single microhole in the nail plate at "initialization" on $h$ at different energies of Yb,Er:Glass laser pulses and $N=50$ are shown in Fig. 3(b). It can be seen that the dependences are not monotonic, but have a pronounced maximum, which depends on the pulse energy. For $E=20 \mathrm{~mJ}$, the maximum $P M=2 \mathrm{mg}$ was observed at $h=0.8 \mathrm{~mm}$; for $E=30 \mathrm{~mJ}$, the maximum $P M=3 \mathrm{mg}$ was also observed at $h=0.8 \mathrm{~mm}$; for $E=40 \mathrm{~mJ}$, the maximum $P M=3 \mathrm{mg}$ was observed at $h=0.6 \mathrm{~mm}$ and for $E=50 \mathrm{~mJ}-$ at $h=0.2 \mathrm{~mm}$ (maximum $P M=1 \mathrm{mg}$ ).

The influence of the distance $H$ from the distal end of the optical fiber to the surface of drug drop on its "initialization" was also investigated. The diameter of laser spot $d_{s}$ in a plane perpendicular to the beam axis and passing through the upper point of the drop, took values $0.83 \div 3.35 \mathrm{~mm}$. It was established that when the optical fiber is at the distance $H=1 \mathrm{~mm}$ up from the drop surface $\left(d_{s}=0.83 \mathrm{~mm}\right)$, the "initialization" of drug delivery through a microhole occurs when applying three Yb,Er:Glass laser pulses with an energy of $60 \mathrm{~mJ}$. 


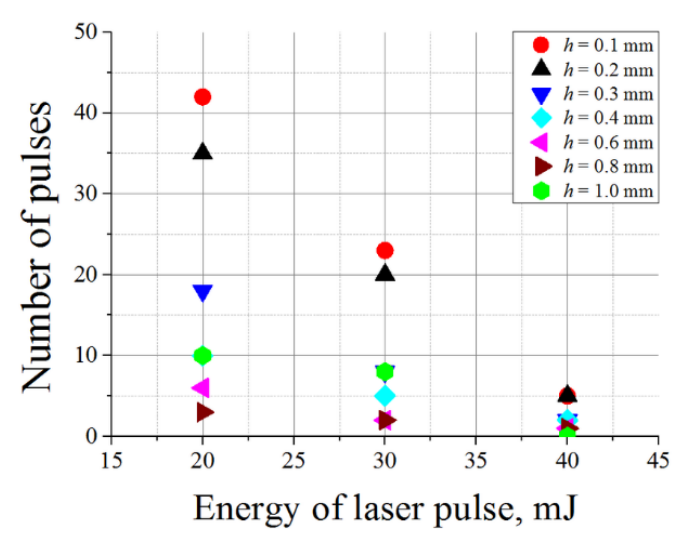

a

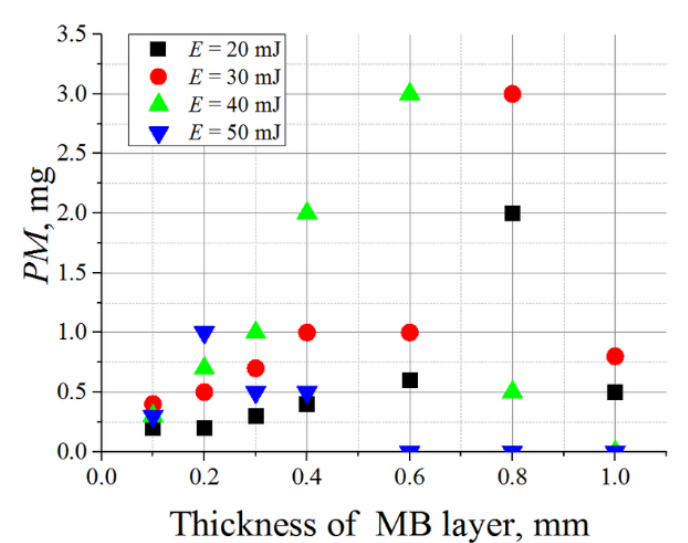

b

Fig. 3 Dependence of the number of pulses necessary for active laser drug delivery from the energy of laser pulse (a) and the mass of the drug penetrated $(P M)$ under the nail plate from the thickness of MB layer $h(\mathrm{~b})$ when using diverging Yb,Er:Glass laser radiation $(H=0.1 \mathrm{~mm}, N=50)$.

When $E=80 \mathrm{~mJ}$ and $100 \mathrm{~mJ}$, "initialization" occurs after just one pulse. When the optical fiber was at the distance $H=5 \mathrm{~mm}$ up from the drop surface $\left(d_{s}=2.27 \mathrm{~mm}\right)$, "initialization" of drug delivery was observed only when applying $100 \mathrm{~mJ}$ Yb, Er:Glass laser pulse, but also after the one laser pulse. When the optical fiber was at $H=8 \mathrm{~mm}\left(d_{s}=3.35 \mathrm{~mm}\right)$, there was no "initialization" of drug delivery under the nail plate.

Similar dependences were obtained during the "initialization" of drug delivery by focused radiation of a Yb,Er:Glass laser (Fig. 4). The number of pulses required for initialization in this case also first decreases with increasing $h$ and then increases (Fig. 4(a)). The fastest initialization was obtained with $h=0.2 \div 0.8 \mathrm{~mm}$ and $E=30 \mathrm{~mJ}$, the number of pulses required for "initialization" in this case was $N=1$.

As for diverging radiation, dependencies of the mass $(P M)$ of the drug penetrated through a single microhole in the nail plate at "initialization" on $h$ at different energies of $\mathrm{Yb}$,Er:Glass laser pulses and $N=50$ (Fig. 4 (b) had maxima but, in this case, these maxima were observed at $h=0.8 \mathrm{~mm}$ for all pulse energies.

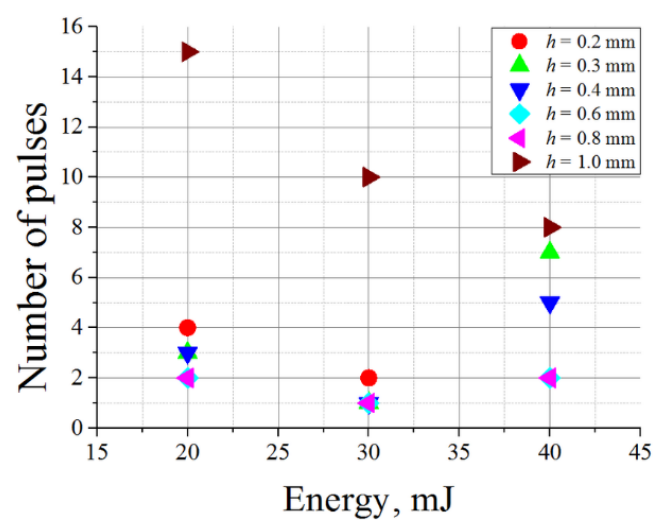

a

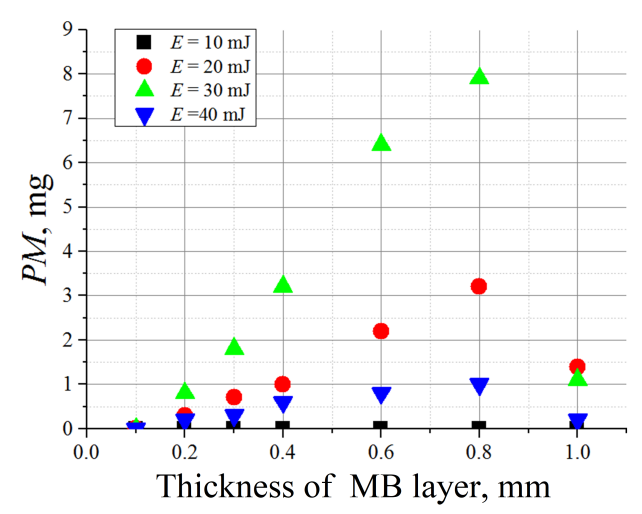

b

Fig. 4 Dependence of the number of pulses necessary for active laser drug delivery from the energy of laser pulse (a) and the mass of the drug penetrated (PM) under the nail plate from the thickness of MB layer $h(\mathrm{~b})$ when using focused Yb,Er:Glass laser radiation $\left(F=11 \mathrm{~mm}, d_{s}=250 \pm 20 \mu \mathrm{m}\right.$, focus on the nail plate surface, $N=50)$.

In Figs. 3(a) and 4(a), the decrease of pulse number $N$ with the increase in laser pulse energy $E$ occurs because with increasing $E$ decreases the number of pulses required to achieve a pressure at the entrance to the microhole sufficient for MB to enter it. The pressure changes as a result of laser-induced heating of MB and the resulting hydrodynamic effects. The magnitude of this pressure is determined by the parameters of laser radiation, the properties of $\mathrm{MB}$, properties of the nail and the size of the microhole. In Figs. 3(b) and 4(b), it is possible to note the presence of a maximum $P M$. In Fig. 3(b), the maximum $P M$ shifts from $h=0.8 \mathrm{~mm}$ to $h=0.2 \mathrm{~mm}$ with increasing $E$ from $20 \mathrm{~mJ}$ to $50 \mathrm{~mJ}$. In Fig. 4(b), the position of the maximum does not depend on $E$ and corresponds to $h=0.8 \mathrm{~mm}$. The shift of $P M$ maximum can be attributed to the MB spatter observed during this experiment, which is associated with the formation of a steam-gas bubble near the MB surface and its exit to the drug surface. The contribution of spatter to the reduction of $P M$ for diverging $\mathrm{Yb}$,Er:Glass laser radiation increases with increasing $E$ and does not depend on $E$ for focused laser radiation. The last one 
can be associated with a significant excess of the power density of the focused laser radiation over the spatter effect threshold. One can also see that $P M$ for focused laser radiation is significantly higher than $P M$ for diverging laser radiation. This effect can also be related to the difference in power densities for both of these modes. The power density of focused laser radiation is greater than the power density of diverging laser radiation, since for focused radiation, $d_{b}=250 \pm 20 \mu \mathrm{m}$, and for the defocused at $H=0.1 \mathrm{~mm}$, the spot diameter is about $500 \mu \mathrm{m}$. It should also be noted that the $P M$ maximum at $h=0.8 \mathrm{~mm}$ correlates with the depth of water absorption of laser radiation with a wavelength of $1.54 \mu \mathrm{m}$.

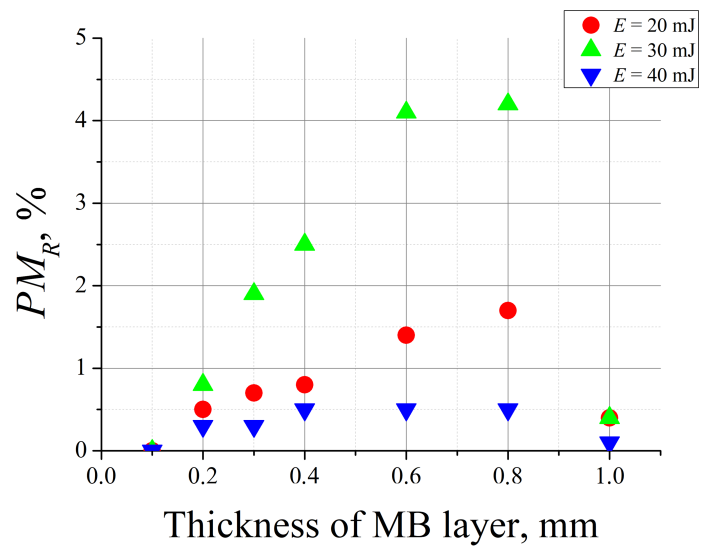

a

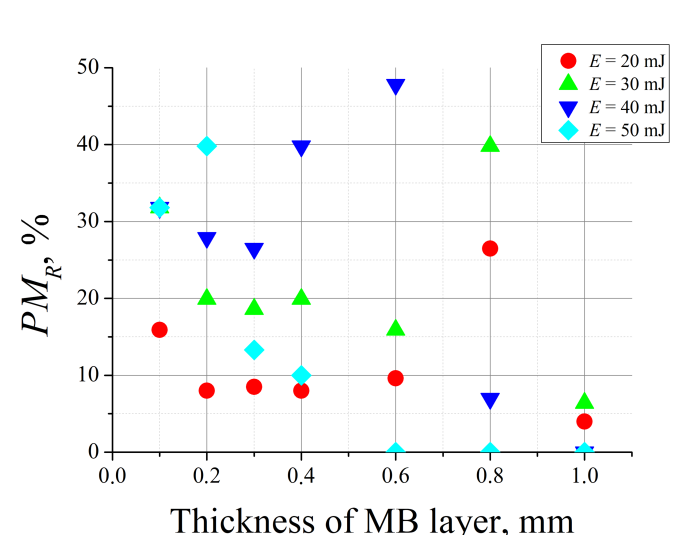

b

Fig. 5 The percentage of $P M$ relative to the mass of the applied drug $\left(P M_{R}\right)$ at different thickness of $\mathrm{MB}$, pulse energies $E$ and $N=50$ when using diverging Yb,Er:Glass laser radiation with $H=0.1 \mathrm{~mm}$ (a) or when using focused on the nail plate surface Yb,Er:Glass laser radiation with $F=11 \mathrm{~mm}$ and $d_{s}=250 \pm 20 \mu \mathrm{m}(\mathrm{b})$.

Fig. 5 shows the percentage of $P M$ relative to the mass of the applied drug $-P M_{R}$. Fig. 5(a) shows the relationship of the mass of the drug applied to the nail plate surface with the $P M$ at different $h$, pulse energies of the diverging $\mathrm{Yb}$,Er:Glass laser radiation, $H=0.1 \mathrm{~mm}$ and $N=50$. Fig, 5(b) shows the relationship $\left(P M_{R}\right)$ of the mass of the drug applied to the nail plate surface with the $P M$ at different $h$ and pulse energies of focused $\mathrm{Yb}$,Er:Glass laser radiation and $N=50$.

It can be seen that the maximum percentage of $P M$ relative to the mass of the drug applied to the nail surface reaches $47.8 \%$ at $h=0.6 \mathrm{~mm}, N=50$ and $E=40 \mathrm{~mJ}$, while the dose of the penetrated drug is $85.7 \pm 10 \mathrm{mg} / \mathrm{cm}^{2}$ and exceeds therapeutically sufficient dose. A further increase in $h$ and energy $E$ (at $h>0.3 \mathrm{~mm}$ ) leads to a decrease in this ratio due to splashing of the drug.

It can be seen that in active delivery by focused Yb,Er:Glass laser radiation, the mass ratio of the applied drug to $P M$ at best (at $E=30 \mathrm{~mJ}$ and $h=0.8 \mathrm{~mm}$ ) reaches $4.2 \%$, which is almost an order of magnitude less than in active delivery by diverging radiation. However, the maximum dose of the penetrated drug in active delivery with focused radiation was $225.7 \pm 15 \mathrm{mg} / \mathrm{cm}^{2}$, which is higher than with active delivery by diverging radiation and also exceeds the therapeutically sufficient dose. This effect is because when exposed to focused radiation, the drug solution is sprayed (splashing) and requires additional application after every two-laser pulses. Splashing of the drug during active delivery by focused Yb,Er:Glass laser radiation can be associated with both a change in the geometry of the laser impact and an increase in power density as a result of a decrease in laser spot diameter

\section{Conclusion}

The features of the active delivery of $0.25 \%$ methylene blue aqueous solution through the nail plate microperforated by Er:YLF laser radiation are investigated under various conditions of excitation of hydrodynamic effects in this solution by Yb,Er:Glass laser radiation. It is established that active delivery is most effective when using diverging Yb,Er:Glass laser radiation $(E=40 \mathrm{~mJ}, h=0.6 \mathrm{~mm}, H=0.1 \mathrm{~mm})$. In this case, the drug is delivered under the nail plate when exposed to a single laser pulse, and within 50 laser pulses $3 \mathrm{mg}$ of solution can be delivered. At the same time $47.8 \%$ of the drug mass applied to the nail surface penetrates the nail plate and the drug dose penetrated under the nail plate is $85.7 \pm 10 \mathrm{mg} / \mathrm{cm}^{2}$. The highest dose of the drug penetrated under the nail plate was fixed when exposed to focused radiation of Yb,Er:Glass laser $(E=30 \mathrm{~mJ}$ and $h=0.8 \mathrm{~mm})$ and amounted to $225.7 \pm 15 \mathrm{mg} / \mathrm{cm}^{2}$.

Thus, in active laser delivery a quantity of the drug, well above the therapeutically sufficient dose, penetrates under the nail plate due to the excitation of hydrodynamic effects by the radiation of $\mathrm{Yb}$,Er:Glass laser.

\section{Disclosures}

All authors declare that there is no conflict of interests in this paper. 


\section{References}

1. L. W. F. Souza, S. V. T. Souza, and A. C. D. C. Botelho, "Distal and lateral toenail onychomycosis caused by Trichophyton rubrum: treatment with photodynamic therapy based on methylene blue dye," Anais brasileiros de dermatologia 89(1), 184-186 (2014).

2. S. Vahabi, R. Fekrazad, S. Ayremlou, and N. Zangeneh, "The effect of antimicrobial photodynamic therapy with radachlorin and toluidine blue on streptococcus mutans: an in vitro study," Journal of dentistry (Tehran, Iran) 8(2), 48-54 (2011).

3. M. G. Strakhovskaya, V. G. Zhukhovitskii, A. F. Mironov, A. M. Seregin, E. F. Stranadko, and A. B. Rubin, "Fungicidal activity of khlorin photosensitizers," Doklady Biochemistry and Biophysics 384(1-6), 155-158, (2002).

4. R. Elkeeb, A. AliKhan, L. Elkeeb, X. Hui, and H. I. Maibach, "Transungual drug delivery: current status," International journal of pharmaceutics 384(1), 1-8 (2010).

5. M. V. Saner, A. D. Kulkarni, and C. V. Pardeshi, "Insights into drug delivery across the nail plate barrier," Journal of drug targeting 22(9), 769-789 (2014).

6. R. M. Adams, "Effects of mechanical trauma on nails," American journal of industrial medicine 8(4-5), 273-280 (1985).

7. S. Murdan, "Enhancing the nail permeability of topically applied drugs," Expert opinion on drug delivery 5(11), 1267-1282 (2008).

8. S. N. Murthy, D. E. Wiskirchen, and C. P. Bowers, "Iontophoretic drug delivery across human nail," Journal of pharmaceutical sciences 96(2), 305-311 (2007).

9. A. Torkar, J. Kristl, and S. Murdan, "Low-frequency ultrasound to enhance topical drug delivery to the nail," Presented at AAPS Annual Meeting and Exposition, San Diego, CA, USA (2007).

10. J. Neev, J. S. Nelson, M. Critelli, J. L. McCullough, E. Cheung, W. A. Carrasco, A. M. Rubenchik, L. B. Da Silva, M. D. Perry, and B. C. Stuart, “Ablation of Human Nail by Pulsed Lasers," Lasers in Surgery and Medicine 21(2), 186-192 (1997).

11. M.-T. Tsai, T.-Y. Tsai, S.-C. Shen, C. Ng, Y.-J. Lee, J.-D. Lee, and C.-H. Yang, "Evaluation of Laser-Assisted Trans-Nail Drug Delivery with Optical Coherence Tomography," Sensors (Basel) 16(12), 2111 (2016).

12. O. O. de Morais, I. M. C. Costa, C. M. Gomes, D. H. Shinzato, G. M. C. Ayres, and R. M. Cardoso, "The use of the Er:YAG 2940nm laser associated with amorolfine lacquer in the treatment of onychomycosis," An Bras Dermatol 88(5), 847-849 (2013).

13. E. B. Abd El-Aal, H. M. Abdo, S. M. Ibrahim, and M. T. Eldestawy, "Fractional carbon dioxide laser assisted delivery of topical tazarotene versus topical tioconazole in the treatment of onychomycosis," Journal of Dermatological Treatment 30(3), 1-6 (2019).

14. A. V. Belikov, A. N. Sergeev, S. N. Smirnov, and A. D. Tavalinskaya, "Microperforation of the human nail plate by radiation of erbium lasers," Frontiers of Optoelectronics 10(3) 299-307 (2017).

15. S. Smirnov, A. V. Belikov, A. V. Skrypnik, A. N. Sergeev, and A. D. Tavalinskaya, "Er: YLF-laser microperforation of the nail plate for drug delivery," Proceedings of SPIE 10716, 107160X (2018).

16. P. Gregorčič, M. Jezeršek, and J. Možina, "Optodynamic energy-conversion efficiency during an Er:YAG-laserpulse delivery into a liquid through different fiber-tip geometries," Journal of Biomedical Optics 17(7), 075006 (2012).

17. M. Frenz, H. Pratisto, F. Konz, E. D. Jansen, A. J. Welch, and H. P. Weber, "Comparison of the effects of absorption coefficient and pulse duration of 2.12-um and 2.79-um radiation on laser ablation of tissue," IEEE Journal of Quantum Electronics 32(12), 2025-2036 (1996).

18. E. D. Jansen, T. Asshauer, M. Frenz, M. Motamedi, G. Delacrétaz, and A. J. Welch, "Effect of pulse duration on bubble formation and laser-induced pressure waves during holmium laser ablation," Lasers in Surgery and Medicine 18(3), 278-293 (1996).

19. A. V. Belikov, S. V. Gagarsky, A. N. Sergeev, and S. N. Smirnov, "Study of hydrodynamic processes in liquids under the influence of powerful microsecond Yb,Er:Glass laser pulses," Journal of Instrument Engineering 60(4), 367-374 (2017) [in Russian].

20. A. V. Belikov, S. V. Gagarsky, A. N. Sergeev, S. N. Smirnov, and A. M. Zagorulko, "Study of acoustic signal during laser hydroacoustic processing of biological tissue by microsecond pulses of ytterbium-erbium glass laser radiation," Scientific and Technical Journal of Information Technologies, Mechanics and Optics 18(6), 939-945 (2018) [in Russian].

21. S. N. Smirnov, S. V. Gagarskiy, Y. Y. Fomicheva, A. V. Belikov, A. N. Sergeev, and A. M. Zagorulko, "Investigation of optical and hydrodynamic processes initiated in biological tissues and liquids under the action of high-power pulses of $1.54 \mu \mathrm{m}$ laser radiation," Proceedings of SPIE 10716, 107160W (2018). 
22. A. V. Belikov, A. M. Zagorulko, and S. N. Smirnov, "Optimum mode for processing cataract lens of the human eye with microsecond pulses of Yb,Er:Glass laser,” Journal of Instrument Engineering 61(8), 734-737 (2018) [in Russian].

23. A. V. Belikov, S. N. Smirnov, A. N. Sergeev, and A. D. Tavalinskaya, "Delivery of the photodynamic agent under the nail plate using Er-laser microperforation and laser-induced hydrodynamic processes," 2018 International Conference Laser Optics (ICLO), 491 (2018).

24. A. V. Belikov, A. V. Skrypnik, K. V. Shatilova, and V. V. Tuchin, "Multi-beam laser-induced hydrodynamic shock waves used for delivery of microparticles and liquids in skin," Lasers in surgery and medicine 47(9), 723-736 (2015).

25. C. Peter, D. Hongwan, A. Küpfer, and B. H. Lauterburg, "Pharmacokinetics and organ distribution of intravenous and oral methylene blue," European journal of clinical pharmacology 56(3), 247-250 (2000). 